\title{
The role of basiliximab in the evolving renal transplantation immunosuppression protocol
}

\author{
Paola Salis \\ Chiara Caccamo \\ Roberto Verzaro \\ Salvatore Gruttadauria \\ Mary Artero
}

Division of Nephrology and Division of Abdominal Transplantation, Istituto Mediterraneo per i Trapianti e Terapie ad Alta Specializzazione, Palermo, Italy

Correspondence: Paola Salis Istituto Mediterraneo per i Trapianti e Terapie ad Alta Specializzazione, Via Ernesto Tricomi, I 90127 Palermo, Italy Tel +390912192111 Fax +390912192400

Email psalis@ismett.edu

\begin{abstract}
Basiliximab is a chimeric mouse-human monoclonal antibody directed against the alpha chain of the interleukin-2 (IL-2) receptor on activated T lymphocytes. It was shown in phase III trials to reduce the number and severity of acute rejection episodes in the first year following renal transplantation in adults and children, with a reasonable cost-benefit ratio. The drug does not increase the incidence of opportunistic infections or malignancies above baseline in patients treated with conventional calcineurin inhibitor-based immunosuppression. In the field of renal transplantation, basiliximab does not increase kidney or patient survival, despite the reduction in the number of rejection episodes. Basiliximab may reduce the incidence of delayed graft function. In comparison with lymphocyte-depleting antibodies basiliximab appears to have equal efficacy in standard immunological risk patients. Recently, IL-2 receptor monoclonal antibodies have been used with the objective of reducing or eliminating the more toxic elements of the standard immunosuppression protocol. Several trials have incorporated basiliximab in protocols designed to avoid or withdraw rapidly corticosteroids, as well as protocols which substitute target-of-rapamycin (TOR) inhibitors for calcineurin inhibitors.
\end{abstract}

Keywords: basiliximab, renal transplantation, IL-2 receptor antagonists, induction, immunosuppression, corticosteroids, calcineurin inhibitors

In the decades that followed the first successful kidney transplant in 1954, the standard immunosuppression protocols for solid organ transplantation have evolved to permit reliable short-term graft survival, such that transplantation has become the preferred therapy for end-stage organ failure. In the case of renal transplantation, graft and patient survival exceed $90 \%$ during the first post-transplant year. Nevertheless, long-term graft survival rates, although improving, have not kept pace with the success of short-term rejection prevention. For recipients of standard deceased donor kidneys performed between 2001 and 2002 unadjusted one-year graft survival was 91\%, and for the period 1997-1998 five-year graft survival was 69\%; for the same time periods, unadjusted five-year graft survival for expanded criteria donors was 51\% (2004 OPTN/SRTR Annual Report). In addition, the pillars of the standard immunosuppression protocols - calcineurin inhibitors, corticosteroids and adjuvant antimetabolites such as azathioprine and mycophenolate mofetil (MMF) - are associated with collateral infectious, neoplastic and metabolic effects which not only lead to organ damage, but also may contribute to the premature patient mortality with a functioning graft that afflicts all organ transplants. Thus, the past decade has been characterized by attempts to modify the standard protocol to maintain excellent shortterm transplant survival, prolong organ half-life, and reduce the dependence on the more toxic immunosuppressants.

The purpose of this review is to describe the role of IL-2 receptor monoclonal antibodies - specifically basiliximab - in the context of this evolving paradigm of 
standard immunosuppression, with an emphasis on renal transplantation. Several useful reviews on the use of basiliximab in renal transplantation are available in the literature (Chapman and Keating 2003; Swiatecka-Urban 2003; Boggi et al 2004; Ramirez and Marino 2007). The main interest of the present work is to demonstrate how basiliximab is currently being used to modify standard calcineurin inhibitor- and corticosteroid-based protocols, particularly from the practical nephrological viewpoint. This review is based on a search of Medline and the Cochrane data bases using the MeSH terms "basiliximab" and "renal transplantation" as of January 30, 2007. The aim of the literature search was to find all randomized controlled studies which incorporated basiliximab in the study protocols. Additional articles were selected on the basis of relevance to specific topics.

\section{Drug development and pharmacological properties}

Several interesting reviews discuss the development of basiliximab as an induction therapy for organ transplantation (Morris and Waldmann 2000; Pascual et al 2001; Church 2003). The T lymphocyte IL-2 receptor is composed of three subunits: a small (55 kD) specific $\alpha$ chain (also known as CD25), a $75 \mathrm{kD} \beta$ chain (CD122) and a $64 \mathrm{kD} \gamma$ chain (CD132) (Morris and Waldmann 2000). The CD25 molecule presented itself as a tempting target for drug development, due to its IL-2 specificity and its presence only on $\mathrm{T}$ cells preactivated with other cytokines, such as IL-1, IL-6, and tumor necrosis factor. Eliminating the cells which bear the CD25 molecule would block downstream events leading to organ rejection, without damaging bystander cells not involved in the rejection process, and therefore reduce unwanted collateral effects - infections and tumors - secondary to over-immunosuppression.

The $\alpha$ subunit of the IL-2 receptor complex was discovered by developing a murine monoclonal antibody against activated T cells, known as anti-Tac (Uchiyama et al 1981). The clinical use of the antibody in animal models, however, was hampered by efficacy defects and side effects. To overcome these problems, the murine variable region of the IgG molecule was fused to the human constant region, thus reducing the immunogenicity of the antibody, prolonging the half-life and improving the interaction with human effector molecules and cells (Queen et al 1989). Two antibodies have been constructed and produced by recombinant DNA techniques from mouse myeloma cell lines: basiliximab, a chimeric monoclonal antibody which includes the entire murine variable region and is approximately $80 \%$ human, and daclizumab, a humanized monoclonal antibody which includes only the hypervariable murine component and is approximately $90 \%$ human. Both drugs are currently on the market with trade names of Simulect (basiliximab, Novartis) and Zenapax (daclizumab, Roche) and have shown virtually the same results in phase III clinical trials, although a randomized, prospective, well-powered study between the two drugs using the currently recommended dosage schedules has not been performed.

Three phase II trials performed in deceased donor kidney recipients (Amlot et al 1995; Kovarik et al 1996; Kovarik et al 1997) demonstrated that basiliximab is not associated with cytokine release phenomena during drug infusion, rarely causes allergic reactions to the murine protein, is endowed with a prolonged half-life in the standard dose, provides effective receptor saturation for 4-6 weeks after transplantation, and pharmacokinetic parameters are not affected by sex or weight in recipients weighing more than 35 kilograms. No treatment benefit was found when activated lymphocytes bearing the IL-2 $\alpha$ chain were suppressed for longer than one month (Amlot et al 1995). Importantly, the phase II data established that serum basiliximab concentrations exceeding $0.2 \mu \mathrm{g} / \mathrm{ml}$ were sufficient to saturate CD25 on circulating $\mathrm{T}$ lymphocytes in vivo. Interestingly, the phase II studies were carried out to determine the antibody dose during the first four to six weeks post-transplant which could achieve this concentration. This dose was found to be $40 \mathrm{mg}$, and it was decided to divide the total dose into two $20 \mathrm{mg}$ subdoses, one infused immediately before surgery and the other administered on day 4 , in order withhold the second dose in case of technical failure in the first days post-transplant (Pascual et al 2001). This simple dosing schedule allows all of the drug to be administered during the hospitalization. In addition, all subsequent clinical trials were based on this dosing schedule, which enhanced reproducibility.

Pharmacokinetic modeling was also performed in the pediatric age group (Kovarik et al 2002); the recommended dosage for patients weighing less than $35 \mathrm{~kg}$ is two doses of $10 \mathrm{mg}$, the first two hours before surgery and the second on day 4, while those patients weighing more than $35 \mathrm{~kg}$ should receive the standard two $20 \mathrm{mg}$ doses. Importantly, it was also shown that cyclosporin blood levels may be significantly increased in children by concomitant administration of basiliximab compared to controls (Strehlau et al 2000). As basiliximab levels declined, there were parallel reductions in the cyclosporin levels, requiring increased doses of the calcineurin inhibitor to maintain therapeutic trough levels.

Also in adults basiliximab may provoke drug interactions with calcineurin inhibitors. Sifontis and colleagues (2002) 
noted significantly elevated tacrolimus trough levels in patients treated with basiliximab during the four days of treatment, compared to controls who received no basiliximab. This observation suggested that basiliximab may induce alterations of the cytochrome P450 pathway in the liver, which is responsible for the metabolism of both cyclosporin and tacrolimus. Nevertheless, because this hypothesized interaction has not been proven, there are no specific recommendations of altering calcineurin inhibitor doses during basiliximab administration. Both mycophenolate mofetil (MMF) and azathioprine also alter basiliximab pharmacokinetics (Kovarik et al 2001); but the clinical consequences of these drug interactions are unclear, and also for these drugs there are no indications for modifying the standard doses of the drugs.

Population pharmacokinetics were again determined during the first large phase III blinded, randomized, placebocontrolled trial in adults (Kovarik et al 1999). The result of the trial in 169 treated patients was that no special populations, based on sex, weight, ethnicity, and proteinuria, were not adequately treated with the standard dosing regimen. Specifically, it was found that the elimination half-life was approximately one week, that hemodialysis did not affect half-life, and on the average receptor-saturating concentrations were maintained for $36 \pm 14$ days. Interestingly, patients who suffered an acute rejection had not cleared basiliximab faster than their counterparts who remained rejection-free to month 6 post-transplant, nor was the duration of CD25 saturation different between the two groups. Saturation duration was not different between groups according to the severity scores of the rejection (Banff grades). The mean duration of IL-2R $\alpha$ saturation in pediatric patients receiving basiliximab $12 \mathrm{mg} / \mathrm{m}^{2}$ of 10 or $20 \mathrm{mg}$, depending on bodyweight, was 31-42 days (Sterkers et al 2000; Kovarik et al 2002).

There are relatively few reports that investigate the pharmacokinetics of basiliximab during extracorporeal renal replacement therapy. Eguchi and colleagues (2004) studied serum levels in a liver transplant recipient who was maintained on continuous hemodiafiltration (CVVHDF using a polymethylmethacrylate filter) throughout the perioperative period until he died on the thirtieth post-operative day. Serum levels of basiliximab remained within the therapeutic range ( $>200 \mathrm{ng} / \mathrm{ml}$ ) at least until post-op day 28, and did not differ from a control patient who was not treated with CVVHDF. CD25 positive cells were appropriately surpressed. However, the concentration of basiliximab is reduced by more than $60 \%$ by a single session of plasmapheresis (Okechukwu et al 2001 ) or nearly $90 \%$ by multiple sessions (Nojima et al 2005).
Massive proteinuria may also significantly reduce antibody concentrations (Nagai et al 2005).

\section{The role of induction therapy in renal transplantation}

We have reviewed how IL-2R antagonists, including basiliximab, have been developed, but before describing the tens of studies that have been performed to determine the efficacy of this class of pharmaceuticals, it may be useful to explain why they were developed, particularly in reference to their role as inducing agents.

In the field of renal transplantation, the term induction refers to a period of intense immunosuppression immediately before and following the implant of the allograft. This intense immunosuppression usually consists of bolus administration of corticosteroids and relatively high dosages of the calcineurin inhibitors - cyclosporin or tacrolimus - to achieve elevated blood concentrations in the perioperative period. These drugs, together with an antiproliferative agent, such as azathioprine or mycophenolate mofetil (MMF), constitute the classic "triple therapy" that now characterizes solid organ transplantation and has led to consistent patient and graft survival rates in the first transplant year, exceeding $90 \%$ for the kidney. The induction period typically lasts 4-6 weeks following transplantation; the dosages of the immunosuppressive drugs are then tapered to lower doses, which constitutes the maintenance phase of immunosuppression, lasting as long as the allograft survives. The rationale for more intense immunosuppression immediately following the transplant procedure derives from the observation that reactive recipient lymphocytes recognize antigen-presenting cells (APCs) of donor origin differently than the recognition that follows when antigen is presented by the recipient's own APCs. When an immune response is mounted against a common pathogen, such as a virus or tumor particle, the antigen must be presented bound to the host's HLA to be recognized by a specific clone of T cells (indirect recognition or HLA restriction). The unique aspect of transplant immunology, on the other hand, is that the recipient's T cells recognize the entire HLA complex of the donor's APCs as foreign (direct recognition), and the brisk rejection reaction that follows results from the fact that many more $\mathrm{T}$ lymphocyte clones with varying specificities - two-per-hundred T cells, in comparison with one-per-ten thousand which respond to common environmental antigens (Auchinloss 1995) — recognize the foreign HLA present on the donor's APCs. With time the donor's APCs are replaced by those of the recipient and perhaps local suppressor phenomena occur which modulate the immune response, and the doses of immunosuppression can be safely lowered. 
Until the introduction of cyclosporin in the mid-1980's, only corticosteroids and azathioprine were available for immunosuppression, and the half-life of the transplanted kidney was approximately 12 months. In 1967 equine antilymphocyte globulin was cautiously introduced as an adjuvant immunosuppressant, despite fears of serum sickness and anaphylactic reactions (Brent 1997). Induction with these early antilymphocyte globulins was associated with fewer rejection episodes; but the production of antilymphocyte globulin was often a local, nonstandardized affair, and the results with these drugs, sometimes very good, were not reproducible between and within transplant centers. The greater efficacy of cyclosporin, which has increased the transplanted kidney half-life several fold, led to the phasing out of these early inducing agents.

According to the Organ Procurement and Transplantation Network (OPTN) data, the use of induction therapy has increased steadily throughout the last decade; $72 \%$ of kidney transplant recipients are now treated with induction immunosuppression, compared to $46 \%$ in 1995 (Meier-Kriesche et al 2006). What, then, has motivated the introduction of new inducing agents in the field of renal transplantation, given the relative success of calcineurin inhibitors? Firstly, not all patient populations have shared in the improved outcomes furnished by standard triple therapy, including those at risk for delayed graft function, highly sensitized patients, AfricanAmericans, patients with chronic hepatitis C or B infections, patients with systemic disease such as diabetes mellitus and others. With the introduction of new immunosuppressants including induction agents, it is becoming possible to tailor the immunosuppressive protocol to the needs of the individual patient. Secondly, after the introduction of standard triple therapy, it soon became clear that collateral effects limited not only graft survival due to nephrotoxicity, but also patient survival was not commensurate with the general population, perhaps due to infectious, oncologic and metabolic effects of the standard immunosuppressants. Thus, it behooved the transplant community to seek ways to reduce or substitute the more toxic elements of the standard protocol, particularly corticosteroids and the calcineurin inhibitors. Finally, the pharmaceutical industry has introduced purification and standardization features to polyclonal antilymphocyte preparations which has increased efficacy and reproducibility, efficacious antiviral prophylaxis which allows the consideration of enhanced immunosuppression, the production of pure humanized monoclonal antibodies such as the IL-2R antagonists with the promise of more targeted immunosuppression and safety, and aggressive marketing.
As mentioned previously, local suppressor phenomena may have a role in dampening the rejection reaction in time. In fact, great interest has been generated by the discovery that naturally arising regulatory $\mathrm{T}$ lymphocytes (Tregs) actively maintain immunological self-tolerance (Sakaguchi 2005). These cells, which are characterized by expression of the surface antigens CD4 and particularly CD25, are associated with a transcription factor, FOXP3, which is necessary for the expression of the key suppressor gene responsible for the $\mathrm{CD} 4^{+} \mathrm{CD} 25^{+}$phenotype. It has been hypothesized that these suppressor $\mathrm{T}$ cells modulate cellular rejection, and that expansion of antigen-specific clones of Tregs may actually lead to peripheral transplantation tolerance. Thus, these important regulatory lymphocytes constitute the small fraction of CD25-positive T cells in the circulation not correlated with immune activation. Could IL-2R antagonists, then, also reduce the population of Tregs during induction therapy, and thus reduce the host's capacity to modulate the rejection response per se? Baan and colleagues (2005) have found that monoclonal anti-CD25 antibodies (in this case, daclizumab) do, in fact, reduce FOXP3 messenger RNA levels in vitro. On the other hand, Game and colleagues (2005) found that the suppressor functions of human $\mathrm{CD} 4{ }^{+} \mathrm{CD} 25^{+} \mathrm{T}$ cells were not diminished in the presence of everolimus or basiliximab - always in vitro - and were, in fact, resistant to the pro-apoptotic effects of the immunosuppressors. The clinical significance of these findings is yet to be determined.

\section{Human efficacy studies}

The success of basiliximab in the phase I and II trials led to two large scale, randomized, blinded, placebo-controlled phase III efficacy trials, one in the United States (Kahan et al 1999) and the other in Europe and Canada (Nashan et al 1997). Both trials studied basiliximab in combination with cyclosporin microemulsion and corticosteroids using standard protocols and drug level monitoring. The principal outcome variable was acute rejection occurring in the first six months and 12 months in the European study and 12 months in the American study. Patient and graft survival were secondary outcome variables. A total of 722 patients were analyzed in the two studies, evenly divided between treated patients and controls, on an intention-to-treat basis. In both studies basiliximab was shown to reduce significantly the incidence of acute rejection at 12 months (in the US study: placebo $54.9 \%$, basiliximab $37.6 \%, \mathrm{p} \leq 0.001$; in the European study: placebo $54.8 \%$, basiliximab $37.9 \%, \mathrm{p} \leq 0.01$ ). Basiliximab was associated with significantly fewer episodes of 
corticosteroid-resistant rejection requiring antilymphocyte therapy, and placebo-treated patients required significantly greater amounts of corticosteroids than basiliximab-treated patients at week 2 . Neither study showed significant differences in graft or patient survival at 12 months. In the US study, a significantly higher proportion of patients produced urine in the operating room compared with patients receiving placebo. Thus, the incidence of delayed graft function was lower in patients treated with basiliximab (15\%) than those treated with placebo $(23 \%, \mathrm{p}=0.07)$, although there were no differences between groups for the need of hemodialysis within the first postoperative week. Also in the US study, the sum of adverse events including graft loss, patient death and rejection was lower in the recipients receiving basiliximab (41.0\% vs $58.4 \%, p=0.001)$. Graft survival was enhanced in African American patients who were treated with basiliximab at six months $(100 \%$ vs $92 \%, p=0.041)$ and at twelve months $(98 \%$ vs $88 \%, \mathrm{p}=0.059)$. In the American study, there was no statistical difference in the incidence of acute rejection between basiliximab and placebo among recipients of live donor kidneys.

Pooled data from the two large phase III trials were used to assess the efficacy of basiliximab in patients with type 1 and type 2 diabetes (Thistlethwaite et al 2000). A total of 80 patients were treated with basiliximab and 70 patients received placebo. Basiliximab treatment was associated with a significant reduction of acute rejection and biopsy-confirmed rejection (by $41 \%$ and $44 \%$, respectively). Basiliximab was also associated with significantly better graft survival compared with placebo ( $96 \%$ vs $86 \%$, $\mathrm{p}=0.022$ ).

Long-term unblinded follow-up data from these two seminal studies have been pooled for the original 722 patients by the manufacturer of basiliximab (Chapman and Keating 2003). Significantly more basiliximab recipients were free from the combined endpoint of death, graft loss or acute rejection at three years, compared with the patients who had been treated with placebo, as assessed by Kaplan Meier curves $(53 \%$ vs $45 \%, \mathrm{p}=0.025)$. However, this difference had equalized by five years following transplantation.

These two phase III efficacy trials were swiftly followed by three additional multicenter, randomized, blinded, placebo-controlled studies in which basiliximab was compared to placebo in patients treated with standard triple immunosuppression including azathioprine (Ponticelli et al 2001; de Boccardo 2002) or mycophenolate mofetil (MMF; Lawen et al 2003). Also in these carefully performed trials, which included a total of 766 patients, basiliximab demonstrated superior prophylaxis of acute rejection compared with placebo, without affecting graft or patient survival rates at six and twelve months. Three-year (Sheashaa et al 2003) and five-year (Sheashaa et al 2005) follow-up of 100 recipients of live donor kidneys is available from a single center, in which patients were randomized to treatment with basiliximab or no induction in combination with standard triple immunosuppression with azathioprine. The results were comparable to the studies cited above: the incidence of acute rejection at five years was reduced in patients treated with basiliximab ( $54 \%$ vs $72 \%, p=0.062)$, but incidence of chronic allograft nephropathy, graft function as assessed by creatinine or creatinine clearance, graft and patient survival did not differ between the two groups after three years. The cumulative steroid dose was lower in patients treated with basiliximab $(\mathrm{p}=0.027)$.

Most of these early studies utilized cyclosporin as the calcineurin inhibitor. Leonardi and colleagues (2004) retrospectively analyzed their results using basiliximab $(\mathrm{n}=51)$ versus no induction $(\mathrm{n}=46)$ in association with dual immunosuppression with tacrolimus and corticosteroids. A significant decrease in acute rejection in the first three months in the basiliximab group ( $2 \%$ vs $17.4 \%, \mathrm{p}<0.01)$ was observed, despite greater immunological risk. Hospital stay was also significantly shorter in the basiliximab group.

\section{Efficacy studies in children}

Several small retrospective studies are available which compare basiliximab with no induction in the pediatric population treated with dual therapy calcineurin inhibitor and corticosteroids (Swiatecka-Urban et al 2001; Vester et al 2001; Pape et al 2002) or triple therapy (Ojogho et al 2005). No statistical differences were observed between groups in terms of acute rejection or graft or patient survival in the 12 months following transplantation, although SwiateckaUrban and colleagues (2001) found a clear advantage when basiliximab was used in patients at high risk for rejection or delayed graft function.

Recently, Grenda and colleagues (2006) reported the results of a prospective, randomized, multicenter trial of basiliximab $(n=99)$ versus no induction $(n=93)$ in a tacrolimus-based protocol that included azathioprine and corticosteroids for standard-risk patients $<18$ years of age who received kidney transplants. Acute rejection rates, steroidresistant rejection rates, patient and graft survival, and renal function were virtually identical between the two groups at six months. Adverse events were similar in both groups in terms of type and frequency, although "toxic nephropathy" 
(apparently tacrolimus-induced) and abdominal pain were observed significantly more frequently in the basiliximab group. Delayed graft function and CMV infections were insignificantly more frequent in the basiliximab group. Offner and colleagues (2006) have also reported the results of a randomized, multicenter, placebo-controlled trial comparing the efficacy and safety of basiliximab with no induction in 202 children, in association with cyclosporin, MMF and corticosteroids. The incidence of biopsy-proven acute rejection at six months was $11 \%$ in the basiliximab group and $16.1 \%$ in the placebo group (NS). The overall incidence of adverse events was also similar in the two groups.

\section{Efficacy in the elderly}

There are relatively few data available to assess the effect of basiliximab on kidney transplant recipients $\geq 60$ years of age. As part of a retrospective study comparing muromonab CD3 vs basiliximab induction and triple therapy, Kode and colleagues (2003) compared the outcome at three months post-transplant in patients younger or older than 60 years of age in those receiving basiliximab. Despite significantly more HLA mismatches and older donor age, the older recipients fared as well as younger patients in terms of acute rejection, severity of rejection, graft survival, patient survival, hospital stay and serum creatinine $(\mathrm{p}=0.13)$. Heifets et al (2004) analyzed their data on 183 consecutive kidney transplant recipients $\geq 60$ years of age over a 12 -year period during which four cohorts could be recognized on the basis of the induction therapy they received: equine antithymocyte globulin $(n=29)$, muromonab CD3 $(n=45)$, basiliximab with corticosteroid maintenance $(n=40)$ and basiliximab without corticosteroid maintenance $(n=69)$. The incidence of acute rejection in the first three months following transplantation was significantly lower in the basiliximab-treated groups (antithymocyte globulin 31\%, muromonab CD3 31\%, basiliximab with steroid maintenance $17.5 \%$ ( $p<0.005$ vs antithymocyte globulin), basiliximab without steroid maintenance $14.5 \%$ ( $p<0.005$ vs antithymocyte globulin and muromonab CD3). Patient and graft survival at one year did not differ significantly between the four groups. The length of hospital stay and mean cost of induction agent were significantly less for the basiliximab groups ( $p$ value not specified).

\section{Efficacy in high-risk groups}

The efficacy and safety of basiliximab induction was assessed in $40 \mathrm{HIV}$-positive patients undergoing renal transplantation under concomitant highly active antiretroviral therapy (Anil Kumar et al 2005). One- and two-year actuarial patient survival was $85 \%$ and $82 \%$, respectively, and graft survival was $75 \%$ and $71 \%$, respectively. The acute rejection rate was $22 \%$. No patient developed AIDS.

Highly sensitized patients - those who have developed high-titer anti-HLA antibodies (indicated by "panel reactive antibodies" [PRA] >20\%) and are considered at elevated risk for allograft rejection - are often treated with induction therapy. Jirasiritham and colleagues (2004) compared their results in 50 high immunological risk patients induced with IL-2R receptor monoclonal antibodies (basiliximab, $\mathrm{n}=27$, or daclizumab, $\mathrm{n}=23$ ) with 196 standard risk patients. The incidence of acute rejection at three years, graft survival and patient survival were not significantly different between the two groups. Tan and colelagues (2005) analyzed their results in 56 patients with $\mathrm{PRA} \geq 30 \%$ who were randomized to basiliximab $(n=36)$ or no induction $(n=20)$. All patients were treated with standard triple therapy with MMF. At three months the incidence of acute rejection was $11.1 \%$ in the basiliximab group and $50 \%$ in the control group ( $\mathrm{p}<0.01$ ); but at 12 months renal function was equivalent, and there were no differences in patient or graft survival. Other studies in highly sensitized patients are discussed below.

\section{Basiliximab vs other inducing agents}

Several studies have compared the efficacy of basiliximab with other inducing agents, including lymphocyte-depleting drugs and daclizumab. Equine antithymocyte globulin (ATG) is uncommonly used nowadays, and the single study in which basiliximab was compared with this drug (Sollinger et al 2001) will not be discussed further, other than to note that the incidence of biopsy-proven rejection was similar between the two groups. Comparing muromonab CD3 and basiliximab, one large study with 271 patients (Pelletier et al 2000) found no differences in acute rejection between treatment groups, but three other studies with a total of 368 patients (Chowdhury et al 2001; Kumar et al 2001; Kode et al 2003) reported that basiliximab was associated with lower incidences of acute rejection than muromonab CD3 and fewer adverse advents. Five-year follow-up is available from one single-center study (Shoskes et al 2006) in which 52 recipients were randomized to receive OKT3 or basiliximab; the incidence of acute rejection was similar between the two groups during the five-year period. There was a trend for greater patient and graft survival in the OKT3 group, although the difference was not statistically significant.

Basiliximab was compared with rabbit ATG plus triple immunosuppression with MMF in a randomized unblinded 
trial in standard-risk (nonsensitized) patients by Lebranchu and colleagues (2002). Cyclosporin microemulsion was added immediately following surgery in the basiliximab group, but its introduction was delayed by $2-10$ days in the rabbit ATG group. There were no differences in the incidence of acute rejection at six and 12 months, although the small group sizes (50 patients/group) precluded a statistical analysis. The incidence of delayed graft function was lower in the rabbit ATG group ( $18 \%$ vs $22 \%$, NS). Five-year follow-up is now available from this study (Al Najjar et al 2006); patient and graft survival were identical. Mean values of serum creatinine at five years were equivalent. A second randomized French study comparing basiliximab and rabbit ATG was performed in 105 standard-risk patients treated with delayed cyclosporin microemulsion, MMF and corticosteroids (Mourad et al 2004). There were no significant differences between groups in the incidence of delayed graft function or acute rejection. Cytomegalovirus (CMV) infection was more common in the patients who received rabbit ATG than in the basiliximab group ( $41.5 \%$ vs $21.2 \%$ ). It should be mentioned that routine prophylaxis against CMV infection was used only in CMV-negative patients who received grafts from CMV-positive donors.

An interesting aspect of this work involved the sequential administration of cyclosporin after the creatinine had fallen to $200 \mu \mathrm{mol} / \mathrm{L}$ or on day 8 in the case of delayed graft function. Delayed graft function (DGF), defined as the need for hemodialysis in the first 3-7 days post-transplant, occurs in approximately $30 \%$ of renal transplant recipients and is associated with significantly increased risk of acute rejection (Sola et al 2004) and increased healthcare costs (Hagenmeyer et al 2004). A strategy commonly used to avoid or ameliorate DGF is to delay or reduce the dose of the calcineurin inhibitor immediately post-transplant, to avoid nephrotoxicity in the kidney recovering from the ischemic insult of cold storage. Conventional wisdom is that IL-2R antagonists should be administered simultaneously with the calcineurin inhibitor, whereas lymphocyte-depleting agents can be used without calcineurin inhibitors in the first days after transplantation to provide adequate immunosuppression without early nephrotoxicity. Nevertheless, in the Mourad study cited above in standard-risk patients, basiliximab also allowed delayed calcineurin inhibitor use without an increase in acute rejection. Pallet and colleagues (2006) performed a retrospective pilot study in which low-dose cyclosporin was introduced sequentially in recipients of expanded criteria donor kidney transplants using rabbit ATG $(n=14)$ or basiliximab $(n=40)$ in association with high-dose MMF and corticosteroids. The incidence of acute rejection was $11.3 \%$ (7.1\% with ATG and $12.6 \%$ with basiliximab). DGF was diagnosed in $54 \%$ of patients $-10(72 \%)$ in the ATG group and $19(48 \%)$ in the basiliximab group $(p=0.03)$. The duration of DGF was longer in the ATG-treated patients $(p=0.03)$. The weight of the evidence as summarized in a complete review of the topic by Sandrini (2005) indicates that IL-2R antagonists can maintain as effective immunosuppression as lymphocyte-depleting agents when calcineurin inhibitor administration is reduced or delayed, including kidneys from older donors, which are at particular risk for DGF (Emparan et al 2003).

In the pediatric age group, Clark and colleagues (2002) compared basiliximab, cyclosporin microemulsion and corticosteroids with an historical control group treated with antilymphocyte globulin, cyclosporin microemulsion, azathioprine and corticosteroids; each group consisted of 42 children who were transplanted consecutively with each immunosuppressive protocol. There were no statistical differences in the incidence of acute rejection or renal function at six months. Fewer CMV infections were observed in the basiliximab group ( $10 \%$ vs $19 \%$, NS), particularly in children exposed to seropositive combinations $(p<0.01)$. In a retrospective analysis from Canada (Acott et al 2001), 23 children treated with basiliximab induction, cyclosporin, azathioprine and corticosteroids were compared to the outcomes in 27 historical controls treated with rabbit ATG or horse antilymphocyte globulin and the same maintenance immunosuppressives. Biopsy-confirmed acute rejection at six months was $9 \%$ in the basiliximab group and $33 \%$ in the lymphocyte-depleting group. Patient and graft survival did not differ between groups.

In a retrospective analysis Haririan and colleagues (2005) compared their results with basiliximab $(n=52)$ or rabbit ATG $(n=36)$ in African American kidney transplant recipients, a group at high risk for allograft rejection and poor graft survival. The groups were not equal for all confounding risk factors; the ATG group tended to have a higher proportion of highly sensitized patients, fewer live donor transplants, younger age, older donor age, and longer duration of dialysis. Nevertheless, after adjusting for these variables, graft outcomes and renal function were virtually equal between the two groups. The overall incidence of acute rejection was lower in the ATG group (14\%) compared with the basiliximab group (29\%), but this difference did not reach statistical significance $(\mathrm{p}=0.10)$.

The largest $(n=278)$ randomized study comparing basiliximab and rabbit ATG in adults was performed in high risk recipients (highly sensitized or at risk for delayed graft 
function) of deceased donor grafts (Brennan et al 2006). All patients were also treated with triple immunosuppression including MMF. The primary outcome variable was a composite of acute rejection, delayed graft function, graft loss, and death. At 12 months there were no statistical differences between groups for the composite endpoint. The rabbit ATGtreated patients had fewer acute rejections ( $15.6 \%$ vs $25.5 \%$, $\mathrm{p}=0.02)$ and required less subsequent antibody therapy for acute rejection $(1.4 \%$ vs $8.0 \%, p=0.005)$, but the incidence of graft loss, delayed graft function and death were the same between groups. The incidences of all adverse events, serious adverse advents (not defined), and cancer were equal between the two groups, whereas infections were more common in the rabbit ATG-treated group (85.8\% vs $75.2 \%, \mathrm{p}=0.03$ ), except for cytomegalovirus (CMV) infection, which in this study was more common in the basiliximab-treated group (17.5\% vs $7.8 \%, p=0.02)$. All patients who had a seropositive CMV status before transplantation and patients who received CMV positive kidneys were treated prophylactically with gancyclovir.

Basiliximab $(n=226)$ was compared retrospectively with alemtuzumab $(n=105)$ in consecutive recipients of simultaneous pancreas-kidney transplants (Sollinger et al 2006); maintenance immunosuppression consisted of tacrolimus, MMF and prednisone in both groups. Freedom from kidney rejection favored the alemtuzumab group (80\% vs $68.8 \%$, $\mathrm{p}=0.0907)$, whereas freedom from CMV infection favored the basiliximab group ( $83.6 \%$ vs $70.7 \%, p=0.0021)$. Patient survival, kidney and pancreas grafts survival, pancreas rejection, and other infectious adverse events including PTLD did not differ between groups. Given the low cost of alemtuzumab (not specified), the authors have chosen alemtuzumab as the induction drug of choice.

\section{Basiliximab as treatment for acute rejection}

The only data regarding the use of basiliximab as antirejection therapy are found in case reports. Eighteen adult solid organ transplant recipients (12 kidney, 2 kidney-pancreas and 4 liver) with acute rejection were treated on an outpatient basis with two doses of basiliximab, with apparent success in 13 patients (Chariat et al 2001). Goh and Lye (2001) administered basiliximab to one kidney transplant recipient with steroid-resistant rejection who refused muromonab CD3 treatment, again apparently with success. Finally, Aw and colleagues (2003) treated seven pediatric liver transplant recipients with steroid-resistant rejection with basiliximab; aspartate aminotransferase levels decreased in five children, who remained rejection-free with a median follow-up of 22 months.

\section{Efficacy data from meta-analyses}

Three meta-analyses of randomized trials have evaluated the efficacy of IL-2 receptor antagonists (basiliximab, daclizumab, murine anti-Tac and others) in renal transplantation (Adu et al 2003; Keown et al 2003; Webster et al 2004). Webster and colleagues (2004) included in the analysis 17 trials in which the inducing agents were compared with placebo $(n=2,786)$ and 15 trials with lymphocyte-depleting agents $(n=1,212)$ through November, 2002. Acute rejection was significantly reduced by $34 \%$ at six months in patients treated with IL-2R antagonists, and these drugs also significantly reduced steroid-resistant rejection. But as mentioned previously in the individual studies, there were no significant differences in graft survival at one and three years. There were no significant differences when the IL2R antagonists were compared with lymphocyte-depleting induction agents in terms of acute rejection. There were also no significant differences in terms of graft loss, patient death, CMV infection or malignancy, but the IL-2R antagonists were associated with fewer episodes of fever, leukopenia and thrombocytopenia. There were no apparent differences between basiliximab and daclizumab in terms of efficacy or side effects.

\section{Drug safety and cost}

Individual placebo-controlled trials and the three metaanalyses of basiliximab efficacy have shown that the drug is well tolerated, always in the context of induction therapy, in the presence of other immunosuppressive agents. According to the meta-analysis by Adu and colleagues (2003), which included 1858 analyzed patients treated with dual or triple cyclosporin-based immunosuppression, there were no significant differences between IL-2R antagonist induction and placebo in terms of overall infections, cytomegalovirus infection, or lymphomas or other malignancies at one year. The meta-analysis by Webster and colleagues (2004) demonstrated that when IL-2R antagonists were compared with polyclonal lymphocyte-depleting agents, there were no significant differences in terms of mortality, CMV infection or malignancy. Fever, leukopenia, and thrombocytopenia were less common in the patients treated with IL-2R antagonists. The data for CMV infections may have been skewed due the large study by Brennan and colleagues (2003; see also previous section). If this trial were removed from the analysis, CMV infections were much less common in patients treated 
with IL-2R antagonists compared with the polyclonals, and these results could not be otherwise explained by baseline immunosuppression, CMV prophylaxis protocol or trial quality. In pediatric renal transplantation recipients Clark and colleagues (2002) reported an insignificant difference in global CMV infection favoring basiliximab compared with antilymphocyte globulin, but these differences were statistically significant in recipients who were seropositive for CMV antibodies $(0 / 16$ vs $8 / 156 ; p<0.01)$. Also in children treated with IL-2R monoclonal antibodies Acott et al (2001) demonstrated a tendency towards fewer infections caused by human herpes virus type 6 and by Epstein Barr virus and a lower incidence of thrombocytopenia and unexplained fever, compared to lymphocyte-depleting antibodies.

Importantly, basiliximab is not associated with the firstdose reactions and cytokine release phenomena that plague muromonab CD3. As mentioned previously, hypersensitivity reactions have been reported with the drug, although they are rare. Postmarketing surveillance revealed 17 cases of hypersensitivity reactions, including anaphylaxis (Baudouin et al 2003). The package insert warns that "extreme caution should be exercised in all patients previously given Simulect when being administered a subsequent course of Simulect." The development of anti-murine antibodies to basiliximab or other murine anti-lymphocyte antibody preparations does not constitute per se a contraindication to the subsequent use of other murine anti-lymphocyte antibody preparations, according to the manufacturer.

Several pharmacoeconomic analyses of basiliximab in comparison with placebo and with other immunosuppressive agents have been performed and are extensively reviewed by Chapman and Keating (2003). In brief, in comparison with placebo, there were no statistically significant differences in costs in terms of immunosuppressive therapies, total hospitalization, laboratory tests, outpatient visits, postoperative dialysis, or total costs at six (Walters et al 2003) or 12 (Chilcott et al 2002; Lorber et al 2000) months. In all the studies cited, basiliximab demonstrated significant reduction in acute rejection without increasing overall treatment costs in comparison to placebo. Chilcott and colleagues (2002) estimated that the cost savings for a single avoided rejection episode in 1996 was US $\$ 9,823$, and six patients in dual therapy needed to be treated with basiliximab to avoid a rejection episode in the seventh patient during the 12 months following renal transplantation; the same group (Walters et al 2003) estimated the cost savings per avoided rejection episode was $£ 1650$ (1997/1998) and that eight patients in triple therapy needed to be treated to avoid acute rejection in the ninth patient after six months.
On the other hand, Crompton and colleagues (2003) found no economic benefit with basiliximab induction in 60 consecutive adult recipients of live donor kidneys in the first 12 months following transplantation. In their retrospective analysis the incidence of acute rejection was $15 \%$ in the control group treated with standard triple therapy without induction and $22 \%$ in the group treated with basiliximab. Mean initial hospitalization charges were significantly higher (US\$51970 vs US\$68,094, 2000, p < 0.05) in the basiliximab group.

\section{Steroid- and calcineurin inhibitor- sparing protocols}

The reduction or elimination of the most toxic elements of the standard protocol - namely corticosteroids and calcineurin inhibitors - constitute the most cogent actual argument in favor of induction therapy for both low-risk and high-risk patients. Approximately 40 published studies can be found in the medical literature from the last decade in which basiliximab induction has been used with this objective.

Aside from their role as immunosuppressors, corticosteroids are associated with a number of hemodynamic and metabolic effects which may shorten the survival not only of the renal allograft, but also generate risk factors which favor cardiovascular disease, the leading cause of mortality in kidney transplant recipients (Vincenti 2004). Among these risk factors are hypertension, diabetes mellitus, and hyperlipidemia. Corticosteroids are also associated with cataract formation, aseptic necrosis of bone, osteoporosis and cosmetic alterations such as acne vulgaris and obesity.

\section{Early steroid withdrawal}

Vincenti and colleagues (2003) randomized 83 low-risk renal transplant recipients to a triple therapy regimen including basiliximab induction, cyclosporin microemulsion, MMF, and standard corticosteroid taper or to a rapid steroid withdrawal protocol in which one dose of methylprednisolone is administered preoperatively and four daily doses postoperatively, followed by complete discontinuation on day 5. There were no significant differences in terms of acute rejection at six (rapid steroid withdrawal 20\% vs standard steroids 9\%) and 12 months (20\% vs 16\%), and there were no differences between groups for corticosteroid-resistant rejection. Graft and patient survival was $100 \%$ at twelve months for the rapid withdrawal group. Seventy-two per cent of the steroid withdrawal group remained off steroids at six months post-transplant. 
It is apparent that the median time to first rejection was shorter for the rapid withdrawal group in comparison to the standard treatment group (31 vs 65 days). This finding may represent an advantage for the basiliximab-treated group, as acute rejection that occurs before hospital discharge or within the first 60 days after transplantation is associated with a reduced risk of chronic allograft nephropathy and improved long-term allograft survival, compared to rejection that occurs after six months (Basadonna et al 1993; Leggat et al 1997).

On the other hand, Woodle and colleagues (2005) performed a multicenter pilot study of rapid steroid withdrawal in 77 standard-risk renal transplant recipients, and suggested that lymphocyte-depleting agents may be more effective for rapid corticosteroid withdrawal recipients, particularly those at high immunological risk, because of early accelerated rejection - before corticosteroids were completely withdrawn - in $7.8 \%$ of patients. Koyama and colleagues (2006) discontinued corticosteroids in 47 patients 14 days after having received $\mathrm{ABO}$-incompatible kidney transplants with basiliximab induction; the results were compared to 15 historical controls treated with standard triple therapy. At six months the incidence of acute rejection, graft survival, patient survival, and mean serum creatinine were equivalent between the two groups. Steroids needed to be resumed in 25 patients $(53.2 \%)$, the motive being acute rejection in 23 patients.

\section{Steroid avoidance}

Xiao and colleagues (2003) compared a protocol consisting of basiliximab, cyclosporin microemulsion and MMF or sirolimus $(n=42)$ with a protocol consisting of the same drugs plus standard corticosteroids $(n=32)$ and found no differences in the incidence of acute rejection at two years after transplantation ( $14 \%$ vs $12 \%)$. There were also no significant differences between the two groups in terms of renal function (as assessed by serum creatinine and creatinine clearance) at one year after transplantation, or graft or patient survival after two years. The same group (Kumar et al 2004) is performing a five-year prospective, randomized, controlled trial in 77 standard-risk patients, and they have reported their interim results at two years. All patients received basiliximab, cyclosporin microemulsion and MMF. Steroids were discontinued after 2-7 days in the rapid withdrawal group and tapered to $5 \mathrm{mg}$ of prednisone by 30 days in the control group. Between groups there were no differences in the rates of acute rejection, subclinical rejection, renal function, or metabolic parameters, although there was a tendency for less new onset diabetes mellitus in the rapid withdrawal group. African Americans fared as well as non-African Americans. Interestingly, a third of the patients in each group received "marginal" kidneys - kidneys acquired from older donors, donors affected by hypertension, diabetes mellitus or mild renal insufficiency - and a tendency towards higher creatinine levels was observed in the rapid withdrawal group compared with controls for the recipients of these kidneys.

A multicenter, randomized three-armed study compared two near-steroid-free protocols - tacrolimus-basiliximab $(\mathrm{n}=153)$ and tacrolimus-MMF $(\mathrm{n}=151)$ - to standard corticosteroids, tacrolimus and MMF ( $\mathrm{n}=147$; Vitko et al 2005) in standard-risk recipients. All patients received a single dose of methylprednisolone $\leq 500 \mathrm{mg}$ perioperatively; the basiliximab-tacrolimus and tacrolimus-MMF groups received no further steroids. The incidence of biopsy-confirmed rejection was significantly higher in the corticosteroid-free groups (basiliximab-tacrolimus 26.1\% and MMF-tacrolimus 30.5\% vs standard therapy $8.2 \%, \mathrm{p}<0.001$ for basiliximab vs standard therapy), and the efficacy of basiliximab and tacrolimus was similar compared to MMF and tacrolimus. The incidence of corticosteroid-resistant rejection, graft survival and patient survival were similar among the three groups. Median creatinine clearance at the end of the study was significantly higher with standard triple therapy $(65.3 \mathrm{ml} / \mathrm{min})$ compared with the corticosteroid-free groups (tacrolimus-MMF 59.4 $\mathrm{ml} / \mathrm{min}$, basiliximab- tacrolimus $55.1 \mathrm{ml} / \mathrm{min}, \mathrm{p}=0.007$ ). The incidence of CMV infection was significantly lower in the basiliximab-tacrolimus group, and there was a tendency for improved metabolic status in this group in terms on newonset diabetes mellitus and reduced mean concentrations of cholesterol.

A randomized, double-blind, multicenter study of 108 renal transplant recipients compared basiliximab to placebo, both in association with cyclosporin microemulsion monotherapy (Parrott et al 2005). The requirement for additional immunosuppression at 12 months post-transplant, the primary endpoint of the study, occurred significantly less frequently in the basiliximab group (54\%) compared with placebo controls $(73 \%, p=0.046)$. The incidence of acute rejection was lower in the basiliximab group, though the difference was not statistically significant (29\% vs $43 \%$, $\mathrm{p}=0.16$ ); graft and patient survival did not differ between groups at 12 months.

Kaufman and colleagues (2005) retrospectively compared their kidney transplant results from 123 patients treated with a single $30 \mathrm{mg}$ dose of alemtuzumab, a 3-day course of methylprednisolone (no oral prednisone), low-dose 
tacrolimus (12-h blood concentrations of 6-8 $\mathrm{ng} / \mathrm{ml}$ ) and MMF to a historical control group of 155 patients in which basiliximab was used for induction with the same maintenance immunosuppressive drugs. Immunological risk was not specified; African Americans comprised about 20\% of each group. During the three-year follow-up, less than $7 \%$ of the recipients required chronic corticosteroid therapy. There were no significant differences in graft and patient survival at one and three years. The incidence of acute rejection at 12 months was $14.9 \%$ for the patients treated with alemtuzumab and $13.5 \%$ for those treated with basiliximab (NS). Rejection in the basiliximab group occurred earlier than those treated with alemtuzumab ( $51 \pm 83$ days post-transplant vs $148 \pm 82$ days). Renal function and type and severity of adverse events were similar between the two groups.

In a prospective, randomized, multicenter trial 335 kidney transplant recipients were randomized to undergo complete steroid avoidance, rapid steroid withdrawal or standard steroids, in association with cyclosporin, enteric-coated mycophenolate sodium and basiliximab induction (Schena et al 2006). At 12 months the primary end-point - glomerular filtration rate - was equivalent in all three groups on an intention-to-treat basis. Biopsy-proven acute rejection was more frequent in the steroid avoidance and rapid withdrawal groups compared to standard steroids $(31.5 \%$ ( $p=0.004$ vs standard steroids), $26.1 \%$ ( $\mathrm{p}=0.046$ vs standard steroids), $14.7 \%$, respectively), and the time to first rejection was also the shorter in these groups. The increment in body mass index and triglyceride levels were significantly lower in the rapid withdrawal group compared to standard steroids (Vincenti et al 2006); fewer patients were treated for hyperglycemia in the steroid avoidance group.

\section{Calcineurin inhibitor withdrawal or avoidance}

Basiliximab has been used in clinical studies with the goal of reducing or eliminating the calcineurin inhibitor from the standard protocol. Hong and Kahan (2001) compared recipients assigned to three treatment groups on the basis of their risk for delayed graft function (DGF). Patients at high risk for DGF were treated with basiliximab, sirolimus, corticosteroids plus low dose cyclosporin microemulsion ( $30 \%$ of the full dose) which was delayed until the resolution of the DGF (creatinine $<2.5 \mathrm{mg} / \mathrm{dl}$; $\mathrm{n}=43$ ) or muromonab CD3 or ATG, corticosteroids, and delayed cyclosporin microemulsion $(n=18)$. Patients at low risk for DGF were treated with basiliximab, corticosteroids and immediate cyclosporin microemulsion $(\mathrm{n}=21)$. After 12 months there were no significant differences in terms of patient or graft survival among the three groups. The mean dose of cyclosporin and corticosteroids was significantly lower for the patients receiving basiliximab and sirolimus than for those in the other two treatment groups ( $p<0.001$ for both comparisons). A retrospective review from the same institution (Knight et al 2004) compared patients who received basiliximab $(n=115)$ or rabbit ATG $(n=30)$ in combination with sirolimus, steroids and delayed low-dose cyclosporin microemulsion. The patients were stratified as high immune responders (African Americans, retransplant recipients or PRA $>50 \%$ ) or low immune responders. The high immune responders treated with basiliximab had a higher incidence of acute rejection episodes $(26 \%)$ than those treated with rabbit ATG $(3 \%, \mathrm{p}=0.01)$ or low responders treated with basiliximab $(10 \%, \mathrm{p}=0.04)$. The investigators also observed a higher mean creatinine level at three months $(\mathrm{p}<0.01$, ANOVA), six months $(\mathrm{p}=0.02)$ and 12 months $(\mathrm{p}=0.01)$ in high responders treated with basiliximab compared to low responders treated with basiliximab or high responders treated with rabbit ATG.

Hamdy and colleagues (2005) randomized 132 live donor kidney transplant recipients to receive low-dose tacrolimus and sirolimus or sirolimus and MMF without the calcineurin inhibitor. Both groups were also treated with basiliximab induction and corticosteroids. The incidence of acute rejection tended to be lower in the calcineurin-free group; there were no differences in graft and patient survival. Renal function after two years was significantly better in the calcineurin-free group, both in terms of serum creatinine $(\mathrm{p}=0.017)$ and calculated creatinine clearance $(\mathrm{p}=0.005)$. Chan and colleagues (2006) randomized 92 patients to receive lower dose vs higher dose tacrolimus in association with everolimus, corticosteroids and basiliximab induction. At six months there were no differences in terms of acute rejection; renal function in terms of creatinine clearance and serum creatinine tended to be better in the low maintenance tacrolimus group. Finally, Martinez-Mier and colleagues (2006) compared a calcineurin inhibitor-free protocol $(n=20)$ consisting of sirolimus, MMF, and corticosteroids to standard triple therapy $(n=21)$ in recipients of live donor kidney transplants in a prospective, randomized, intention-to-treat trial. Basiliximab was used as induction therapy in the sirolimus group $(n=8)$ and the cyclosporin group $(n=3)$ only for patients with greater than three HLA mismatches. None of the patients who received basiliximab and who maintained sirolimus levels in the target range had acute rejection after 12 months. One patient in the cyclosporin group had an acute 
rejection (p NS). No significant difference in mean serum creatinine were noted after 12 months; the sirolimus group had significantly higher cholesterol and triglyceride levels.

\section{Conclusions}

Basiliximab induction significantly reduces the risk of acute cellular rejection in the 12 months following renal transplantation in standard risk adult recipients at a cost equivalent to conventional therapy in deceased donor transplants. However, in well-powered, carefully controlled trials and in the three meta-analyses, the drug did not improve patient or graft survival in standard risk patients in the short- or in the long-term. Particularly in pediatric patients and in recipients of kidneys from live donors, basiliximab has not conferred any advantage in terms of rejection prophylaxis or cost.

This review has cited the principal prospective randomized controlled trials in which basiliximab was incorporated in the study protocol. However, we also included interesting smaller prospective and retrospective studies performed in patient groups which may eventually benefit from anti-CD25 immunosuppression. In high immunologic risk recipients treated with otherwise conventional therapy, basiliximab substantially reduces the incidence of acute rejection compared with no induction. It seems to be less effective than lymphocyte-depleting antibodies in reducing acute rejection in high-risk patients in the short-term, although it is associated with fewer infectious and hematological side effects. However, clinical evidence for this group of patients is still limited, and a longer period of follow-up may be required to discern a clear advantage for one induction strategy over the other.

Basiliximab provides adequate immunosuppression to allow sequential administration of calcineurin inhibitors. Larger prospective studies are needed in older patients and in recipients of marginal kidneys to confirm the reduction of delayed graft function found in the small retrospective studies; the same groups of patients appear to fare less well with steroid withdrawal, which needs to be further studied. Also, we need to confront the issue of IL-2 monoclonal antibodies in the treatment of acute rejection. Although these pharmaceuticals are approved only for induction, it is clear that they are also sporadically used for fragile patients with rejection, particularly children.

The current role for basiliximab in the evolution of the renal transplant immunosuppressive protocol, however, appears to be as induction when corticosteroids are to be withdrawn rapidly following transplantation or when the calcineurin inhibitor is to be administered at reduced doses or avoided in association with a TOR inhibitor in low- and standard-risk patients. Although a worthy goal, the data from these small studies is equivocal at best. Rejection is more frequent in the steroid withdrawal and avoidance groups despite basiliximab induction. Although rejection occurs early following transplantation and should be amenable to treatment, it is unclear if renal function will be compromised in the long-term. No definite conclusion can currently be drawn regarding calcineurin inhibitor reduction or elimination using basiliximab induction because the studies are too few and the group sizes are too small. The future of IL-2 monoclonal antibodies in the immunosuppressive armamentarium will depend in part in defining those patient characteristics which allow avoidance of the most toxic elements of the standard protocol.

\section{Acknowledgments}

We thank Ms Ignazia Di Salvo for assistance in preparation of the manuscript, Mr Filippo La Spesa, Mr Giorgio Damiani, Mr Giuseppe Caracausi, Mr Manlio Cirafici for technical assistance, and Prof Bruno Gridelli for critical evaluation.

\section{References}

Acott PD, Lawen J, Lee S, et al. 2001. Basiliximab versus ATG/ALG induction in pediatric renal transplants: comparison of herpes virus profile and rejection rates. Transplant Proc, 33:3180-3.

Adu D, Cockwell P, Ives NJ, et al. 2003. Interleukin-2 receptor monoclonal antibodies in renal transplantation: meta-analysis of randomised trials. BMJ, 326:789-91.

Al Najjar A, Etienne I, Le Pogamp P, et al. 2006. Long-term results of monoclonal anti-IL2-receptor antibody versus polyclonal antilymphocyte antibodies as induction therapy in renal transplantation. Transplant Proc, 38:2298-9.

Amlot PL, Rawlings E, Fernando ON, et al. 1995. Prolonged action of a chimeric interleukin-2 receptor (CD25) monoclonal antibody used in cadaveric renal transplantation. Transplantation, 60:748-56.

Anil Kumar MS, Sierka DR, Damask AM, et al. 2005. Safety and success of kidney transplantation and concomitant immunosuppression in HIVpositive patients. Kidney Int, 67:1622-9.

Auchinloss H Jr 1995. Antigen presentation. In: Bach FH, Auchinloss H Jr ed. Transplantation Immunology. New York: Wiley-Liss, pp. 95-8.

Aw MM, Taylor RM, Verma A, et al. 2003. Basiliximab (Simulect) for the treatment of steroid-resistant rejection in pediatric liver transplant recipients: a preliminary experience. Transplantation, 75:796-9.

Baan CC, van der Mast BJ, Klepper M, et al. 2005. Differential effect of calcineurin inhibitors, anti-CD25 antibodies and rapamycin on the induction of FOXP3 in human T cells. Transplantation, 80:110-17.

Basadonna GP, Matas AJ, Gillingham KJ, et al. 1993. Early versus late acute renal allograft rejection: impact on chronic rejection. Transplantation, 55:993-5.

Baudouin V, Crusiaux A, Haddad E, et al. 2003. Anaphylactic shock caused by immunoglobulin E sensitization after retreatment with the chimeric anti-interleukin-2 receptor monoclonal antibody basiliximab. Transplantation, 76:459-63.

Boggi U, Danesi R, Vistoli F, et al. 2004. A benefit-risk assessment of basiliximab in renal transplantation. Drug Safety, 27:91-106.

Brennan DC. 2003. Thymoglobulin versus Simulect for induction immunosuppression in cadaveric renal transplant recipients: expanded results from a prospective, randomized, multicenter trial. Am J Transplant, 5(Suppl 3):438-9. 
Brennan DC, Daller JA, Lake KD, et al. 2006. Rabbit antithymocyte globulin versus basiliximab in renal transplantation. $N$ Engl J Med, 355:1967-77.

Brent L. 1997. A History of Transplantation Immunology. San Diego: Acad Pr, Inc. pp. 319-20.

Chan L, Cooper M, Leventhal J, et al. 2006. Excellent renal function at 6 months in de novo kidney transplant recipients treated with everolimus, basiliximab and reduced tacrolimus exposure. Transplantation, 82(Suppl 3):299-300.

Chapman TM, Keating GM. 2003. Basiliximab: a review of its use as induction therapy in renal transplantation. Drugs, 63:2803-35.

Chariat MN, Erren M, Chariat M, et al. 2001. Basiliximab in the therapy of acute rejection after organ transplantation. Transplant Proc, 33:2380.

Chilcott JB, Holmes MW, Walters S, et al. 2002. The economics of basiliximab (Simulect) in preventing acute rejection in renal transplantation. Transplant Int, 15:486-93.

Chowdhury S, Kode RK, Ranganna K, et al. 2001. Induction strategy using basiliximab combined with mycophenolate MMF and immediate lowdose cyclosporin is steroid sparing and more effective than OKT3. Transplant Proc, 33:1057-8.

Church AC. 2003. Clinical advances in therapies targeting the interleukin-2 receptor. $Q J$ Med. 96:91-102.

Clark G, Walsh G, Deshpande P, et al. 2002. Improved efficacy of basiliximab over antilymphocyte globulin induction therapy in paediatric renal transplantation. Nephrol Dial Transplant, 17:1304-9.

Crompton JA, Somerville T, Smith L, et al. 2003. Lack of economic benefit with basiliximab induction in living related donor adult renal transplant recipients. Pharmacother. 23:443-50.

de Boccardo G. 2002. Latin American study of the efficacy and safety of Simulect in kidney transplant recipients. Transplantation, 74:467.

Eguchi S, Yanaga K, Okudaira S, et al. 2004. Immunodynamics of basiliximab in liver allograft recipient under continuous hemodiafiltration. Transplantation, 77:1477-8.

Emparan C, Laukötter M, Wolters H, et al. 2003. Calcineurin-free protocols with basiliximab induction allow patients included in "old-to-old" programs to achieve standard kidney transplant function. Transplant Proc, 35:1326-7.

Game DS, Hernandez-Fuentes MP, Lechler RI. 2005. Everolimus and basiliximab permit suppression by human $\mathrm{CD} 4^{+} \mathrm{CD} 25^{+}$cells in vitro. Am J Transplant, 5:454-64.

Goh HK, Lye WC. 2001. Biopsy-proven resolution of steroid-resistant acute rejection with basiliximab therapy in a renal allograft recipient. Transplant Proc, 33:3213-14.

Grenda R, Watson A, Vondrak K, et al. 2006. A prospective, randomized, multicenter trial of tacrolimus-based therapy with or without basiliximab in pediatric renal transplantation. Am J Transplant, 6:1666-72.

Hagenmeyer E-G, Haussler B, Hempel E, et al. 2004. Resource use and treatment costs after kidney transplantation: impact of demographic factors, comorbidities and complications. Transplantation, 77:1545-50.

Hamdy AF, El-Agroudy AE, Bakr MA, et al. 2005. Comparison of sirolimus with low-dose tacrolimus versus sirolimus-based calcineurin inhibitor-free regimen in live donor renal transplantation. Am J Transplant, 5:2531-8.

Haririan A, Morawski K, Sillix DH, et al. 2005. Induction therapy with basiliximab versus thymoglobulin in African-American kidney transplant recipients. Transplantation, 79:716-21.

Heifets M, Saeed MI, Parikh MH, et al. 2004. Induction immunosuppression in kidney transplant recipients older that 60 years of age: safety and efficacy of ATGAM ${ }^{\circledR}$, OKT3 ${ }^{\circledR}$ and Simulect ${ }^{\circledR}$. Drugs Aging, 21:747-56.

Hong JC, Kahan BD. 2001. A calcineurin antagonist-free induction strategy for immunosuppression in cadaveric kidney transplant recipients at risk for delayed graft function. Transplantation, 71:1320-8.

Jirasiritham S, Sumethkul V, Mavichak V, et al. 2004. The role of anti-IL2 receptor in high-risk kidney transplant patients. Transplant Proc, $36: 2110-12$

Kahan BD, Rajagopalan PR, Hall M. 1999. Reduction of the occurrence of acute cellular rejection among renal allograft recipients treated with basiliximab, a chimeric anti-interleukin-2-receptor monoclonal antibody. United States Simulect Renal Study Group. Transplantation, 67:276-84.
Kaufman DB, Leventhal JR, Axelrod D, et al. 2005. Alemtuzumab induction and prednisone-free maintenance immunotherapy in kidney transplantation: comparison with basiliximab induction - long-term results. $\mathrm{Am}$ $J$ Transplant, 5:2539-48.

Keown PA, Balshaw R, Khorasheh S, et al. 2003. Meta-analysis of basiliximab for immunoprophylaxis in renal transplantation. Biodrugs, 17:271-9.

Knight RJ, Kerman RH, Schoenberg L, et al. 2004. The selective use of basiliximab versus thymoglobulin in combination with sirolimus for cadaveric renal transplant recipients at low risk versus high risk for delayed graft function. Transplantation, 78:904-10.

Kode R, Fa K, Chowdhury S, et al. 2003. Basiliximab plus low-dose cyclosporin vs OKT3 for induction immunosuppression following renal transplantation. Clin Transplant, 17:369-76.

Kovarik JM, Rawlings E, Sweny P, et al. 1996. Pharmacokinetics and immunodynamics of chimeric IL-2 receptor monoclonal antibody SDZ CHI 621 in renal allograft recipients. Transplant Int, 9(Suppl 1):S32-33.

Kovarik J, Wolf P, Cisterne JM, et al. 1997. Disposition of basiliximab, an interleukin-2 receptor monoclonal antibody, in recipients of mismatched cadaver renal allografts. Transplantation, 64:1701-5.

Kovarik JM, Kahan BD, Rajagopalan PR, et al. 1999. Population pharmacokinetics and exposure-response relationships for basiliximab in kidney transplantation. Transplantation, 68:1288-94.

Kovarik JM, Pescovitz MD, Sollinger HW, et al. 2001. Differential influence of azathioprine and mycophenolate mofetil on the disposition of basiliximab in renal transplant patients. Clin Transplant, 15:123-30.

Kovarik JM, Offner G, Broyer M, et al. 2002. A rational dosing algorithm for basiliximab (Simulect) in pediatric renal transplantation based on pharmacokinetic-dynamic evaluations. Transplantation, 74:966-71.

Koyama I, Fuchinone S, Iwado K, et al. 2006. Steroid can be safely withdrawn in ABO-incompatible kidney transplant recipients by using basiliximab. Transplantation, 82(Suppl 3):516.

Kumar AMS, Fa K, Vankawala R, et al. 2001. Simulect, calcineurin inhibitor, mycophenolate mofetil, and prednisone is more effective than OKT3, calcineurin inhibitor, mycophenolate mofetil, and prednisone in African American kidney recipients in reducing acute rejections and prolonging graft survival. Transplant Proc, 33:3195-6.

Kumar MSA, Xiao S-G, Fyfe B, et al. 2004. Steroid avoidance in renal transplantation using basiliximab induction, cyclosporine-based immunosuppression and protocol biopsies. Clin Transplant, 19:61-9.

Lawen JG, Davies EA, Mourad G, et al. 2003. Randomized double-blind study of immunoprophylaxis with basiliximab, a chimeric anti-interleukin-2 receptor monoclonal antibody, in combination with mycophenolate mofetil-containing triple therapy in renal transplantation. Transplantation, 75:37-43.

Lebranchu Y, Bridoux F, Buchler M, et al. 2002. Immunoprophylaxis with basiliximab compared with antithymocyte globulin in renal transplant patients receiving MMF-containing triple therapy. Am J Transplant, 2:48-56.

Leggat JE, Ojo AO, Leichtman AB, et al. 1997. Long-term renal allograft survival: prognostic implication of the timing of acute rejection episodes. Transplantation, 63:1268-72.

Leonardi G, Messina M, Giraudi R, et al. 2004. Basiliximab in association with tacrolimus and steroids in caucasian cadaveric renal transplanted patients: significant decrease in early acute rejection rate and hospitalization time. Clin Transplant, 18:113-18.

Lorber MI, Fastenau J, Wilson D, et al. 2000. A prospective economic evaluation of basiliximab (Simulect $\left.{ }^{\circledR}\right)$ therapy following renal transplantation. Clin Transplant, 14:479-85.

Martinez-Mier G, Mendez-Lopez MT, Budar-Fernandez LF, et al. 2006. Living related kidney transplantation without calcineurin inhibitors: initial experience in a Mexican center. Transplantation, 82:1533-6.

Meier-Kriesche H-U, Li S, Gruessner RWG, et al. 2006. Immunosuppression: evolution in practice and trends, 1994-2004. Am J Transplant, 6(Part 2):1111-31.

Morris JC, Waldmann TA. 2000. Advances in interleukin 2 receptor targeted treatment. Ann Rheum Dis 59(Suppl I):i109-14. 
Mourad G, Rostaing L, Legendre C, et al. 2004. Sequential protocols using basiliximab versus anti-thymocyte globulins in renal-transplant patients receiving mycophenolate mofetil and steroids. Transplantation, 78:584-90.

Nagai T, Goto Y, Haba T, et al. 2005. The effect of urinary protein excretion in post-renal transplant recurrent nephrotic syndrome on basiliximab pharmacokinetics and pharmacodynamics in a pediatric patient. Transplant Proc, 37:879-80.

Nashan B, Moore R, Amlot P, et al. 1997. Randomised trial of basiliximab versus placebo for control of acute cellular rejection in renal allograft recipients. CHIB 201 International Study Group. Lancet, 350(9086):1193-8. (Erratum in: Lancet, 1997. 350(9089):1484.)

Nojima M, Yoshimoto T, Nakao A, et al. 2005. Sequential blood level monitoring of basiliximab during multisession plasmapheresis in a kidney transplant recipient. Transplant Proc, 37:875-8.

Offner G, Hoecker B, Pape L, et al. 2006. A multicenter, placebo controlled trial evaluating the efficacy and safety of basiliximab $\left(\right.$ Simulect $\left.{ }^{\mathbb{R}}\right)$ in combination with CsA, MMF and steroids in pediatric renal allograft recipients, 12 months results. Transplantation 82(Suppl 3):99.

Ojogho O, Sahney S, Cutler D, et al. 2005. Mycophenolate mofetil in pediatric renal transplantation: non-induction vs induction with basiliximab. Pediatr Transplant, 9:80-3.

Okechukwu CN, Meier-Kriesche H-U, Armstrong D, et al. 2001. Removal of basiliximab by plasmapheresis. Am J Kidney Dis, 37:E11.

Pallet N, Anglicheau D, Martinez F, et al. 2006. Comparison of sequential protocol using basiliximab versus antithymocyte globulin with highdose mycophenolate mofetil in recipients of a kidney graft from an expanded-criteria donor. Transplantation, 81:949-52.

Pape L, Strehlau J, Henne T, et al. 2002. Single centre experience with basiliximab in paediatric renal transplantation. Nephrol Dial Transplant, 17:276-80.

Parrott NR, Hammad AQ, Watson CJE, et al. 2005. Multicenter, randomized study of the effectiveness of basiliximab in avoiding addition of steroids to cyclosporine A monotherapy in renal transplant recipients. Transplantation, 79:344-8.

Pascual J, Marcén R, Ortuño J. 2001. Anti-interleukin-2 receptor antibodies: basiliximab and daclizumab. Nephrol Dial Transplant, 16:1756-60.

Pelletier RP, Davies EA, Elkhammas EA, et al. 2000. Improved outcome in renal transplant recipients treated with Simulect versus OKT3 induction. Transplantation, 69:S157.

Ponticelli C, Yussim A, Cambi V, et al. 2001. A randomized, double-blind trial of basiliximab immunoprophylaxis plus triple therapy in kidney transplant recipients. Transplantation, 72:1261-7.

Queen C, Schneider WP, Selick HE, et al. 1989. A humanized antibody that binds to the interleukin-2 receptor. Proc Natl Acad Sci USA, 86:10029-33.

Ramirez CB, Marino IR. 2007. The role of basiliximab induction therapy in organ transplantation. Expert Opin Biol Ther, 7:137-48.

Sakaguchi S. 2005. Naturally arising Foxp3-expressing CD $25^{+} \mathrm{CD} 4{ }^{+}$regulatory $\mathrm{T}$ cells in immunological tolerance to self and non-self. Nature Immunol, 6:345-52.

Sandrini S. 2005. Use of IL-2 receptor antagonists to reduce delayed graft function following renal transplantation: a review. Clin Transplant, 19:705-10.

Schena FP, Vincenti F, Paraskevas S, et al. 2006. 12-month results of a prospective, randomized trial of steroid avoidance, steroid withdrawal and standard steroids in de novo renal transplant patients receiving cyclosporine, enteric-coated mycophenolate sodium (EC-MPS, myfortic $\left.^{\circledR}\right)$ and basiliximab. Transplantation, 82(suppl 3):84-5.

Sheashaa HA, Bakr MA, Ismail AM, et al. 2003. Basiliximab reduces the incidence of acute cellular rejection in live related donor kidney transplantation: a three-year prospective randomized trial. J Nephrol, 16:393-8.

Sheashaa HA, Bakr MA, Ismail AM, et al. 2005. Long-term evaluation of basiliximab induction therapy in live donor kidney transplantation: a five-year prospective randomized study. Am J Nephrol, 25:221-5.

Shoskes DA, Flechner SM, Shoskes VL, et al. 2006. A randomized prospective trial of OKT3 vs basiliximab induction in renal transplantation with over 5 year follow up. Transplantation, 82(Suppl 3):494.
Sifontis NM, Benedetti E, Vasquez EM. 2002. Clinically significant drug interaction between basiliximab and tacrolimus in renal transplant recipients. Transplant Proc, 34:1730-2.

Sola R, Alarcon A, Jimenez, et al. 2004. The influence of delayed graft function. Nephrol Dial Transplant, 19(Suppl 3):iii32-7.

Sollinger H, Kaplan B, Pescovitz MD, et al. 2001. Basiliximab versus antithymocyte globulin for prevention of acute renal allograft rejection. Transplantation, 72:1915-19.

Sollinger HW, Odorico JS, Becker YT, et al. 2006. Campath vs basiliximab after simultaneous pancreas-kidney transplantation in 331 patients. Transplantation, 82(Suppl 3):103.

Sterkers G, Baudouin V, Ansart-Pirenne H, et al. 2000. Duration of action of a chimeric interleukin-2 receptor monoclonal antibody, basiliximab, in pediatric kidney transplant recipients. Transplant Proc, 32:2757-9.

Strehlau J, Pape L, Offner G, et al. 2000. Interleukine-2 receptor antibodyinduced alterations of ciclosporin dose requirements in paediatric transplant recipients. Lancet, 356:1327-8.

Swiatecka-Urban A, Garcia C, Feuerstein D, et al. 2001. Basiliximab induction improves the outcome of renal transplants in children and adolescents. Pediatr Nephrol, 16:693-6.

Swiatecka-Urban A. 2003. Anti-interleukin-2 receptor antibodies for the prevention of rejection in pediatric renal transplant patients: current status. Pediatr Drugs, 5:699-716.

Tan J, Yang S, Wu W. 2005. Basiliximab (Simulect) reduces acute rejection among sensitized kidney allograft recipients. Transplant Proc, 37:903-5.

Thistlethwaite JR Jr, Nashan B, Hall M, et al. 2000. Reduced acute rejection and superior 1-year renal allograft survival with basiliximab in patients with diabetes mellitus. The Global Simulect Study Group. Transplantation, 70:784-90.

Uchiyama T, Nelson DL, Fleisher TA, et al. 1981. A monoclonal antibody (anti-Tac) reactive with activated and functionally mature human T-cells. J Immunol, 126:1398-403.

Vester U, Kranz B, Testa G, et al. 2001. Efficacy and tolerability of interleukin-2 blockade with basiliximab in pediatric renal transplant recipients. Pediatr Transplant, 5:297-301.

Vincenti F, Monaco A, Grinyo J, et al. 2003. Multicenter randomized prospective trial of steroid withdrawal in renal transplant recipients receiving basiliximab, cyclosporin microemulsion and mycophenolate mofetil. Am J Transplant, 3:306-11.

Vincenti F. 2004. Interleukin-2 receptor antagonists and aggressive steroid minimization strategies for kidney transplant patients. Transplant Int, 17:395-401

Vincenti F, Schena FP, Paraskevas S, et al. 2006. Metabolic effects of steroid avoidance or early steroid withdrawal: 12-month results of a randomized trial in de novo renal transplant patients receiving cyclosporine, enteric-coated mycophenolate sodium (EC-MPS) and basiliximab. Transplantation, 82(Suppl 3):483.

Vitko S, Klinger M, Salmela K, et al. 2005. Two corticosteroid-free regimens - tacrolimus monotherapy after basiliximab administration and tacrolimus/mycophenolate mofetil - in comparison with a standard triple regimen in renal transplantation: results of the Atlas Study. Transplantation, 80:1734-41.

Walters SJ, Whitfield M, Akehurst RL, et al. 2003. Economic implications of the use of basiliximab in addition to triple immunosuppressive therapy in renal allograft recipients: a UK perspective. Pharmacoeconomics, 21:129-38.

Webster AC, Playford EG, Higgins G, et al. 2004. Interleukin 2 receptor antagonists for renal transplant recipients: a meta-analysis of randomized trials. Transplantation, 77:166-76.

Woodle ES, Vincenti F, Lorber MI, et al. 2005. A multicenter pilot study of early (4-day) steroid cessation in renal transplant recipients under Simulect, tacrolimus and sirolimus. Am J Transplant, 5:157-66.

Xiao SG, Lee D, Fyfe B, et al. 2003. Two-year patient and graft survival, graft function and chronic allograft nephropathy with steroid-free Neoral based immunosuppression in kidney transplant recipients - a controlled study. Am J Transplant, 5(3 Suppl):440. 